\title{
Allergie und Asthma durch Fast Food
}

Der steigende Konsum von Fast Food beschert diversen Arztgruppen vermehrten Zulauf. Unter ihnen sind möglicherweise auch Pneumologen und Allergologen.

Die Prävalenz von Asthma und atopischen Erkrankungen hat über die vergangenen Jahrzehnte drastisch zugenommen. Lungenfachärzte aus China und Australien glauben, einen möglichen Grund dafür aufgedeckt zu haben: die Zunahme des Fast-FoodVerzehrs. In einer Übersichtsarbeit [1] konnten sie sogar einen dosisabhängigen Zusammenhang mit dem Asthmarisiko feststellen.

In der Analyse wurden 16 Studien 13 Querschnitts- und drei Fall-KontrollStudien - aus den Jahren 2001 bis 2015 mit Teilnehmerzahlen zwischen 140 und 500.000 berücksichtigt. Bei FastFood-Konsumenten war das Risiko, an Asthma zu leiden, um $58 \%$, und das Risiko für ein schweres Asthma um $34 \%$ erhöht. Ihr Wheezing-Risiko lag um $21 \%$ über dem von Menschen, die auf Fertigprodukte für den schnellen Verzehr verzichteten. Alle Unterschiede waren statistisch signifikant.

\section{Mehr Burger, mehr Asthma}

Darüber hinaus wurde auch für andere allergische Erkrankungen ein Zusammenhang mit dem Verzehr von Fast Food ermittelt: für ärztlich diagnostizierte allergische Rhinitis (relatives Risiko $+43 \%$ ), schwere Ekzeme (+51\%), Rhinokonjunktivitis (+21\%) und schwere Rhinokonjunktivitis (+54\%) (• Abb. 1).

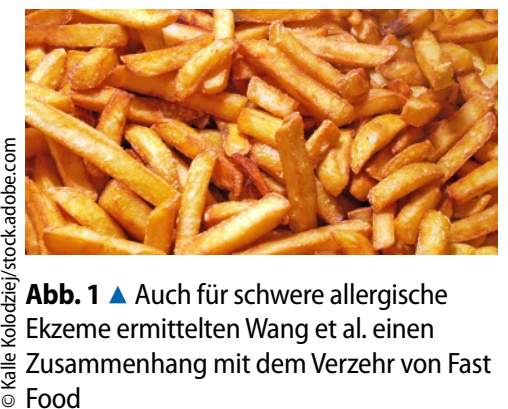

In den Studien zu Asthma bzw. Wheezing und Fast Food war außerdem eine Dosis-Wirkungs-Beziehung zu erkennen. So war etwa das Risiko eines schweren Asthmas um $9 \%$ erhöht, wenn ein- bis zweimal wöchentlich Lebensmittel wie Burger, Pommes oder Döner verspeist wurden, es lag aber um $34 \%$ höher, wenn das mindestens dreimal in der Woche der Fall war.

Soweit die Art des Fast Food genauer spezifiziert war, war vor allem die Menge an wöchentlich konsumierten Burgern mit dem Risiko für Asthma oder Wheezing assoziiert. Bei zuckerhaltigen Softdrinks oder Takeaway-Essen ließ sich keine Korrelation erkennen.

\section{Übergewicht und Entzündung als mögliche Mediatoren}

Eine Schwäche des Reviews besteht darin, dass die Qualität der Studien insgesamt niedrig war und dass es keine einheitliche Definition von Fast Food gab. Generell zählen dazu in Masse produzierte, schnell zubereitete Lebensmittel, die typischerweise reichlich Kalorien, gesättigte und Transfette, Zucker, einfache Kohlenhydrate und Kochsalz enthalten.

Die Verbindung zwischen Junk Food und Asthma könnte zum Teil über den Anstieg des BMl vermittelt sein. Bei Adjustierung für den BMI war nämlich nur noch im Fall-Kontroll-Design ein signifikanter Anstieg des Asthmarisikos zu sehen.

Darüber hinaus könnten aber auch direkte Effekte von Burger und Co. zur Asthmaentstehung beitragen, wie die Studienautoren um Cheng S. Wang von der Universität in Chengdu ausführen. Zum Beispiel stimulieren gesättigte Fette über die Aktivierung von Toll-like-

\section{Das Wichtigste in Kürze}

Gibt es einen Zusammenhang zwischen Fast-Food-Verzehr und Asthma? Fast-Food-Konsumenten haben häufiger Asthma und atopische Erkrankungen.

Bedeutung: Möglichst wenig Burger und sonstiges Fast Food zu essen, könnte im Hinblick auf Asthma und atopische Erkrankungen günstige Effekte haben.

Einschränkung: Der Anstieg des Asthmarisikos könnte über die Zunahme des BMI vermittelt sein. Die ausgewerteten Studien erlauben keine Aussage über einen kausalen Zusammenhang.

Rezeptoren die Freisetzung von proinflammatorischen Zytokinen.

Wang und Kollegen haben selbst in einer Untersuchung gezeigt, dass durch fettreiche Mahlzeiten die Atemwegsentzündung bei Asthma verschlimmert wird. Außerdem könnte der mit dem Fast-Food-Konsum verbundene Verzicht auf gesündere Lebensmittel nachteilig wirken. Auch dazu gibt es eine Studie von Wang et al., der zufolge bei einem Speiseplan mit wenig Obst und Gemüse das Risiko für Asthmaexazerbationen zunimmt.

\section{Literatur}

1. Wang SC et al (2018) Is the consumption of fast foods associated with asthma or other allergic diseases? Respirology

hautnah $2018 \cdot 17: 120$

https://doi.org/10.1007/s12326018-0305-5

(c) Springer-Verlag GmbH Austria, ein Teil von Springer Nature 2018 